Antikörper Aducanumab, dem Gammasekretase-Inhibitor Avagacestat, den Betasekretase-Inhibitoren E2609 und Verubecestat, dem Betaamyloid-Antikörper Gantenerumab und dem Betaamyloid-Aggregationshemmer KHK6640.

Auch Präventionsstudien mit Antikörpern in Biomarker-definierten Populationen laufen: eine Studie mit drei Therapiearmen (Solanezumab, Ganterenumab und dem Betasekretaseinhibitor LY2886721) bei Patienten mit genetischen Mutationen, die zu einer familiären $\mathrm{AD}$ führen, eine weitere zur passiven Immunisierung mit dem Anti-Amyloid-Antikörper Crenezumab bei Patienten mit einer genetischen Mutation für eine frühe familiäre AD in Kolumbien und eine Studie mit Solanezumab bei symptomatischen älteren gesunden Menschen ohne genetisches Risiko mit nachgewiesener $A \beta$-Ablagerung im Amyloid-PET.

\section{Rekrutierung gestaltet sich schwierig}

In der Praxis gestaltet sich die Rekrutierung solcher Studienteilnehmer aber schwierig, wie Frölich an einem Beispiel zeigte: Über die eigene Gedächtnisambulanz wurden für eine solche
Studie 189 Patienten geprüft, gerade sieben erfüllten die geforderten Kriterien. Von den über ein Callcenter ermittelten 57 Kandidaten entsprachen nur 23 den Einschlusskriterien und die über regionale Medien angesprochenen 26 potenziellen Kandidaten reduzierten sich aufgrund der Einschlusskriterien auf 14. Die weitere Prüfung der potenziellen Studienteilnehmer führte schließlich zu einer tatsächlichen Rekrutierung von nur fünf Patienten. „Diese Patientenpopulation ist dann sehr homogen nach den Ein- und Ausschlusskriterien, aber extrem selektiert", bedauerte Frölich. So ließen sich Patienten mit MCI für solche Studien zwar abgrenzen, für die Beratung unselektierter Patienten im Alltag sei das aber nicht anwendbar. Im Übrigen ist er der Überzeugung: Ein therapeutischer Ansatz alleine wird nicht den Durchbruch bringen.

Friederike Klein, freie Medizinjournalistin

Symposium: Kompetenznetz Demenzen: Klinische Forschung im Kontext der neuen Diagnosekriterien für Demenzerkrankungen, Vortrag Lutz Frölich, Mannheim: Kausale Therapie: Wie früh muss man behandeln, um erfolgreich zu sein? DGPPN-Kongress 2015, Berlin, 25.-28.11.2015

\title{
Subjektive Parameter können bei der Demenzvorhersage helfen
}

\author{
Die Erkennung früher Zeichen einer sich ankündigen- \\ den Alzheimer-Demenz (AD) ist Voraussetzung dafür, \\ Therapiestudien in einer frühen Erkrankungsphase \\ oder gar zur Prävention bei Hochrisikopersonen \\ durchzuführen. Subjektive Beschwerden können \\ dabei prognostisch hilfreich sein.
}

Derzeit geht man davon aus, dass der Beginn der Demenzentstehung bei der Alzheimer-Erkrankung 20 bis 30 Jahre vor der diagnostisch relevanten kognitiven Leistungsbeeinträchtigung liegt. Ein prodromales Stadium einer AD kann nach der International Working Group (IWG) diagnostiziert werden bei einem spezifischen klinischen Phänotyp mit

- episodischen Gedächtnisstörungen,

- einer berichteten oder beobachteten kognitiven Verschlechterung über sechs Monate und

- einem objektivierbaren Gedächtnisdefizit vom hippokampalen Typ nach einem spezifischen Test,

wenn gleichzeitig ein In-vitro-Nachweis von

- reduziertem Amyloid-beta 42 (A $\beta 42)$ und erhöhtem Tau oder gTau im Liquor

- oder ein Amyloid-Nachweis im PET

- oder eine nachgewiesene dominante, kausale Mutation vorliegt [Dubois B et al. Lancet Neurol 2014; 13: 614-29].

Ein spezifischer Test für das Gedächtnisdefizit vom hippokampalen Typ ist der „Free and Cued Selective Reminding Test“ (FCSRT), in dem zunächst Objekte anhand Abbildungen gelernt werden, diese später frei erinnert werden sollen und anschließend Hinweise (cues) gegeben werden, um fehlende zu erinnern. „Patienten mit Depression können durch diese Hinweise weitere Objekte erinnern, Demenzpatienten nicht“, erläuterte Professor Frank Jessen, Direktor der Universitätsklinik für Psychiat- rie in Köln. Eine Untersuchung des Kompetenznetzes Demenzen zeigte, dass der FCSRT das hippokampale Defizit widerspiegelt und auch mit pathologischen Liquorparametern assoziiert ist. Er ist allerdings nicht sensitiv genug für eine Früherkennung: Von 185 Patienten mit leichter kognitiver Beeinträchtigung (MCI), die einen unauffälligen, normwertigen FCSRT zeigten, wiesen $62,7 \%$ pathologisch veränderte Liquor-Biomarker auf.

\section{Was der Patient sagt, ist wichtig}

Sorgen um die eigene Gedächtnisleistung (Subjective Concerns of Demantia, SCD) korrelieren aber in Schwere und Art mit den pathologischen Liquormarkern nicht nur bei MCI, sondern auch bei Prä-MCI [Wolfsgruber S et al. Neurology 2015; 84: $1261-8]$. „Man muss gar nicht warten, bis jemand MCI hat“, meinte Jessen und betonte: „Entgegen der Meinung von vor einigen Jahren ist das, was der Patient sagt, wichtig“. Bei Patienten mit SCD war das Risiko, in drei darauffolgenden Jahren ein MCI oder eine AD zu entwickeln, nach einer noch nicht publizierten Studie des Kompetenznetzes mit 82 Patienten bei gleichzeitigem Nachweis von pathologischen A $\beta 42$-Werten im Liquor um mehr als das Doppelte (Hazard Ratio 2,2) und bei Vorliegen von pathologischen Werten von $A \beta 42$ und Tau um mehr als das Dreifache (Hazard Ratio 3,1) erhöht. „Es ist aber viel zu früh, um jetzt alle mit Sorgen um ihr Gedächtnis zu punktieren“, räumte Jessen ein. Zunächst müsse genauer herausgearbeitet werden, welche Art von Gedächtnisbedenken wirklich relevant sind.

Friederike Klein, freie Medizinjournalistin

Session: Prädiktion der Alzheimer-Erkrankung, Vortrag Frank Jessen, Köln: Neuropsychologische und subjektive Prädiktoren der Alzheimer-Demenz. DGPPN-Kongress 2015, Berlin, 25.-28.11.2015 